\title{
Die Arbeitsgemeinschaft Onkologische Bildgebung in der Deutschen Krebsgesellschaft
}

Die Stimme der Bildgebung in der Deutschen Krebsgesellschaft ist die Arbeitsgemeinschaft Bildgebung in der Onkologie (ABO). Die ABO wird abwechselnd von einem Mitglied der Deutschen Röntgengesellschaft und der Deutschen Gesellschaft für Nuklearmedizin geleitet. Turnusgemäß wurde auf dem diesjährigen 31 . Deutschen Krebskongress (19.22.02.2014, Berlin) ein Vertreter der DRG gewählt. Neuer Vorsitzender der ABO ist ab sofort Prof. Heinz-Peter Schlemmer aus Heidelberg. Er ist zudem Vorsitzender der Arbeitsgemeinschaft Onkologische Bildgebung der DRG. Als stellvertretender Vorsitzender wurde Prof. Jörg Kotzerke, Direktor der Klinik und Poliklinik für Nuklearmedizin, gewählt.

Im Rahmen des Krebskongresses organisierte die AG eine Sitzung, auf der den Onkologen und Patienten neue Entwicklungen auf dem Gebiet der Bildgebung vorgestellt wurden. Prof. Zöphel aus Dresden und Prof. Schlemmer zeigten in diesem Rahmen Vorträge zur PET-CT in der Lymphomdiagnostik. Die Sitzung bot die einmalige Möglichkeit, den Zuhörerkreis direkt mit Themen anzusprechen, die der Bildgebung am Herzen liegen. Als AG in der Sektion $B^{*}$ stehen Forschungsgelder zur Verfügung, die in Absprache innerhalb der AG relativ frei eingesetzt werden können.

\section{Werden Sie Mitglied der ABO! $\nabla$}

DRG-Mitglieder, die auch Mitglied der Deutschen Krebsgesellschaft sind, können genauso Mitglied der AG Bildgebung in der Onkologie der Deutschen Krebsgesellschaft werden. Wir möchten Sie bitten, Mitglied dieser wichtigen AG zu werden, denn die Mittelzuweisung an die ABO hängt von der Anzahl der Mitglieder ab!

Prof. Schlemmer unterstützt gerne Ihren Antrag.

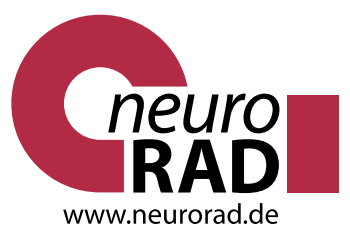

